\title{
Driving force for commensurate vortex domain formation in periodic pinning arrays
}

\author{
S.J. Bending ${ }^{a, *}$, A.N. Grigorenko ${ }^{b}$, M.J. Van Bael ${ }^{c}$, M. Lange ${ }^{c}$, \\ V.V. Moschalkov ${ }^{\text {c }}$, H. Fangohr ${ }^{\text {d }}$, P.A.J. de Groot ${ }^{\mathrm{d}}$ \\ a Department of Physics, University of Bath, Claverton Down, Bath BA2 7AY, UK \\ ${ }^{\mathrm{b}}$ Department of Physics and Astronomy, University of Manchester, Manchester M13 9PL, UK \\ ${ }^{\mathrm{c}}$ Laboratorium voor Vaste-Stoffysica en Magnetisme, Katholieke Universiteit Leuven, Celestijnenlaan 200D, 3001 Leuven, Belgium \\ d School of Engineering Sciences \& Department of Physics and Astronomy, University of Southampton, Southampton SO17 1BJ, UK
}

\begin{abstract}
Recent vortex images in periodic pinning arrays have revealed the formation of degenerate commensurate domains separated by domain walls near rational fractional filling. This phenomenon was entirely unanticipated since, in stark contrast to ferromagnetic materials, the energies and magnetisation of different domains are identical, and the driving force for domain formation and estimation of typical domain sizes has, until now, remained an unsolved problem. We use high-resolution scanning Hall probe microscopy to show that domain formation is driven by the efficient incorporation of mismatched excess vortices/vacancies at the corners of domain walls. Molecular dynamics simulations with a generic pinning potential reveal that domains are only formed when vortex-vortex interactions are long range. A semi-quantitative model of domain formation further discloses how domain sizes depend on both the pinning array period and effective penetration depth.
\end{abstract}

(C) 2004 Elsevier B.V. All rights reserved.

Keywords: Superconducting vortices; Pinning arrays; Commmensurate states

\section{Introduction}

Recent advances in nanofabrication and thin film growth technologies have made it possible to engineer many of the properties of superconducting materials. An important example is the enhancement of the critical current of a superconductor by introducing a periodic artificial pinning array. It is

\footnotetext{
* Corresponding author. Tel.: +44-1225-385173; fax: +441225-386110.

E-mail address: pyssb@bath.ac.uk (S.J. Bending).
}

now well known that commensurate vortex structures form in these materials at certain specific values of the vortex density when sharp peaks in the bulk magnetisation and critical current are observed [1-7]. Many different types of artificial pinning object have been successfully realised including arrays of sub-micron holes (antidots) [1-3] or ferromagnetic dots with both in-plane [4,5] and perpendicular anisotropy [6,7], yet the qualitative features of the matching phenomena appear to be generic to all of these. The strongest critical current enhancements occur at integer matching fields, 
when one or more vortices become trapped at each pinning site. Much weaker effects are, however, also seen at fields corresponding to rational fractional filling. Simulations originally indicated that these correspond to simple ordered vortex structures which are commensurate with the underlying pinning array but where the occupation of each pinning site was not necessarily the same [8]. There have been several high-resolution studies of commensurate structures in these systems using Lorentz [9] and scanning Hall probe (SHPM) $[10,11] \mathrm{mi}-$ croscopies. Our understanding of the magnetisation process near integer matching fields is now quite advanced. Critical current enhancements are believed to arise from the formation of a multi-terrace critical state whereby a staircase of terraces of different integer matching fields exists with very high screening currents flowing at the inter-terrace boundaries $[12,13]$. No such understanding exists at fractional matching fields, however, where two or more possible degenerate commensurate structures always exist under the symmetry transformations of the pinning array. Indeed recent SHPM images by Field et al. [10] have revealed the spontaneous formation of complex composite structures of degenerate domains separated by domain walls near rational fractional filling. This phenomenon was entirely unanticipated since, in stark contrast to ferromagnetic materials, the energies and magnetisation of different domains are identical, and the driving force for domain formation and the estimation of typical domain sizes has, until now, remained an unsolved problem. Here we show that domain formation is intimately related to the longrange vortex-vortex interactions in thin superconducting films. Under these conditions domain wall corners act as sinks for excess vortices or vacancies and greatly extend the field range over which commensurate states can occur [14].

\section{Experimental method}

Our experiments were performed on a $50 \mathrm{~nm}$ granular $\mathrm{Pb}$ film $\left(T_{\mathrm{c}}=7.17 \mathrm{~K}\right)$ deposited over an $a=1 \mu \mathrm{m}$ period square array $\left(H_{1}=\Phi_{0} / a^{2}=\right.$ $20.67 \mathrm{Oe}$ ) of $0.4 \mu \mathrm{m}$ square ferromagnetic 'dots' with perpendicular magnetic anisotropy patterned in a $[\operatorname{Co}(0.3 \mathrm{~nm}) / \operatorname{Pt}(1.1 \mathrm{~nm})]_{10}$ multilayer film $[5,7,15]$. The overall array size was $\sim 2 \mathrm{~mm} \times 2$ $\mathrm{mm}$ and contained about $4 \times 10^{6}$ magnetic dots. The magnetic dots exhibit rather square magnetisation loops with large coercive fields $(\sim 1 \mathrm{kOe})$, and make ideal pinning 'templates' for studying matching effects in the adjacent superconducting film at the much lower relevant applied fields $(0-$ 50 Oe). Pearl has shown [16] that vortex-vortex interactions are long-range in a thin film whose thickness $t \ll \lambda$. Under these circumstances the interaction energy decays as $\sim \log _{\mathrm{e}}(\Lambda / r)$ for $r \ll \Lambda(T)$ and $\sim 1 / r$ for $r \gg \Lambda(T)$, where $\Lambda(T)=$ $2 \lambda^{2}(T) / t$ is called the effective penetration depth. For our $\mathrm{Pb}$ film $(t=50 \mathrm{~nm})$ we estimate that $\Lambda(T)=2 \lambda^{2}(T) / t=92 \mathrm{~nm} /\left(1-T / T_{\mathrm{c}}\right)$ from measurements of the upper critical field of a comparable reference film.

We have used high-resolution scanning Hall probe microscopy (SHPM) as sketched in Fig. 1(a)

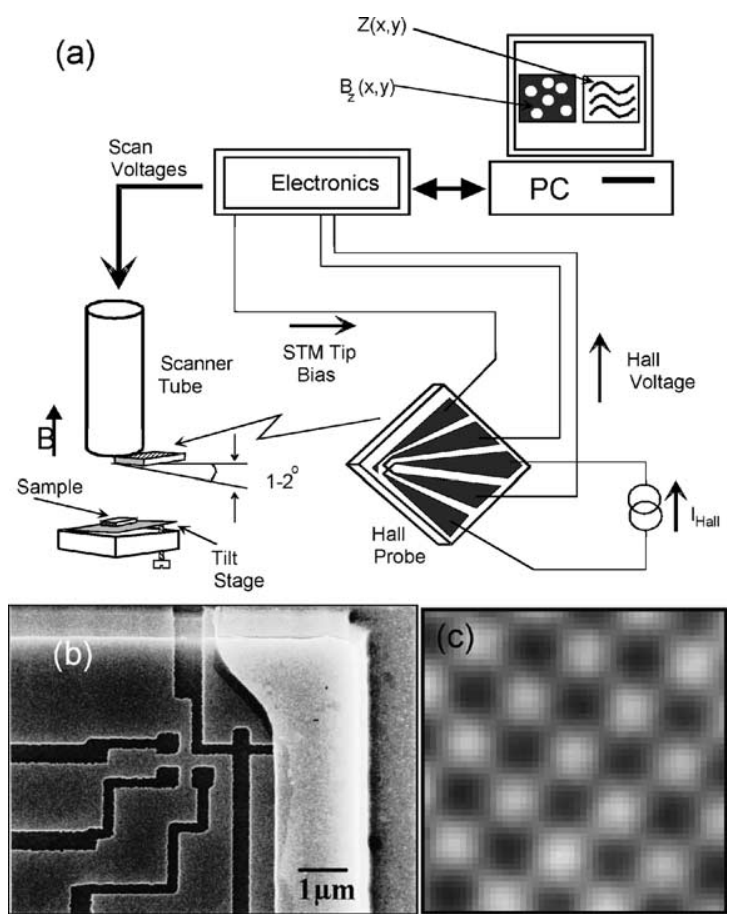

Fig. 1. (a) Schematic diagram of the Scanning Hall probe microscope. (b) Scanning electron micrograph of a sub-micron scanning Hall probe with integrated STM tip. (c) Image of magnetised pinning array at $T>T_{\mathrm{c}}(\mathrm{Co} / \mathrm{Pt}$ dots are black). 
to both image the magnetisation state of the $\mathrm{Co} / \mathrm{Pt}$ nanomagnets, and to visualise discrete vortices trapped on them. Our SHPM is a modified commercial low temperature STM in which the usual tunnelling tip has been replaced by a microfabricated GaAs/AlGaAs heterostructure chip as illustrated in Fig. 1(b). Electron beam lithography and wet etching were used to define a Hall probe in the two-dimensional electron gas at the intersection of two $\sim 400 \mathrm{~nm}$ wide wires approximately $5 \mu \mathrm{m}$ from the corner of a deep mesa etch. The latter had been coated with a thin Au layer to act as an integrated STM tip. The sample is first approached towards the sensor until tunnelling is established and then retracted about $100 \mathrm{~nm}$ allowing rapid scanning with minimum detectable fields $\sim 1 \mu \mathrm{T} / \mathrm{Hz}^{0.5}$. The Hall probe makes an angle of about $1^{\circ}$ with the sample plane so that the STM tip is always the closest point to the surface, and the Hall sensor was about $300-400 \mathrm{~nm}$ above the sample in the images shown here. A more detailed description of the instrument is given elsewhere [17].

\section{Experimental results}

Prior to any imaging experiments the pinning array was magnetised $(|H|>1 \mathrm{kOe})$ such that the moments pointed downwards with respect to the Hall scanner. Fig. 1(c) shows an image of the magnetised pinning array above the critical temperature of the superconducting film, where every black spot corresponds to the location of a $\mathrm{Co} / \mathrm{Pt}$ dot. This magnetic template was retained for all subsequent experiments.

Local magnetisation data collected with the Hall probe parked a few microns above the sample reveal a remarkable asymmetry. With the field applied in the same direction as the dot magnetic moments strong irreversibility is observed with pronounced matching features, while the magnetisation collapses almost to zero in the opposite direction. High-resolution SHPM images of the vortex structures formed after field-cooling to below $T_{\mathrm{c}}$ revealed that this arises because vortices whose fields are parallel to the $\mathrm{Co} / \mathrm{Pt}$ dot magnetic moments $(H<0)$ are attracted to them and become strongly pinned, while those with anti-par- allel fields $(H>0)$ are repelled into interstitial spaces and are weakly pinned. This is illustrated in Fig. 2 which shows images of the vortex structure formed at (a) $H / H_{1}=+1 / 2$ and (b) $H / H_{1}=-1 / 2$. In both cases we see a relatively well-ordered 'checkerboard' structure with one vortex present for every two unit cells of the pinning array. However, for the antiparallel orientation (a) they are repelled to interstitial spaces while in the parallel orientation (b) the vortices are pinned at $\mathrm{Co} /$ Pt dots. The possible reasons for this asymmetry are discussed in [15].

Fig. 3(a)-(d) show a typical family of images for a sample cooled at fields slightly above and below $-H_{1} / 2$. Remarkably this did not result in excess vortices/vacancies in the commensurate lattice as is the case at field values close to integer matching [11]. Instead coexisting domains of the two degenerate commensurate states are observed separated by domain walls of varying complexity (indicated by the superimposed lines). The latter appear to move smoothly as slightly different cooling fields are employed and their presence does not seem to be related to the possible existence of inhomogeneities in the pinning array. There is, however, a crucial difference between the two sets of images. For $|H|<H_{1} / 2$, when vacancies should exist in the checkerboard structure, the corners where domain walls bend by $90^{\circ}$ comprise a 'low density' square cluster of three unoccupied pins and one occupied one. In contrast, for $|H|>H_{1} / 2$, when excess vortices should exist, the corners are composed of a 'high density' cluster of three
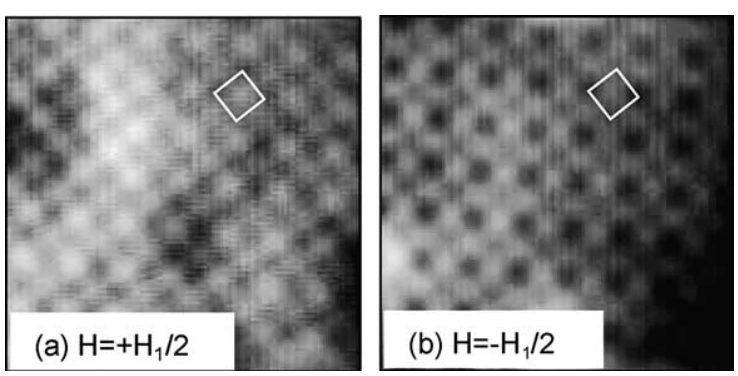

Fig. 2. Vortex 'checkerboard' structure after field-cooling to 6.8 $\mathrm{K}$ in (a) $H=+H_{1} / 2$ (white vortices) and (b) $H=-H_{1} / 2$ (black vortices). In each case one unit cell of the pinning array is indicated. 

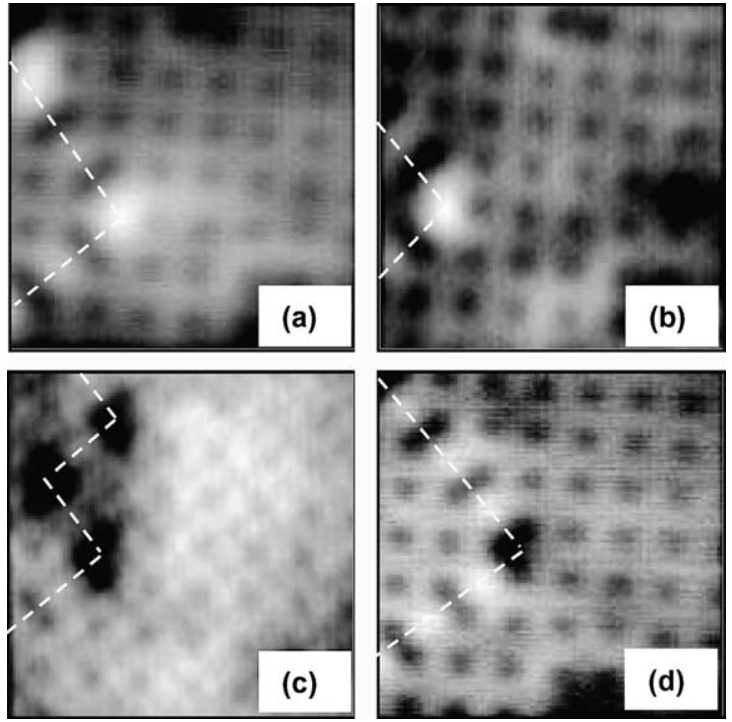

Fig. 3. SHPM images of commensurate vortex configurations after field-cooling close to the half-matching field: (a) $H=$ $-0.990 \cdot H 1 / 2, T=5.5 \mathrm{~K}$; (b) $H=-0.997 \cdot H 1 / 2, T=5.5 \mathrm{~K}$; (c) $H=-1.010 \cdot H 1 / 2, T=6.8 ; \mathrm{K}$ (d) $H=-1.04 \cdot H 1 / 2, T=$ $6.8 \mathrm{~K}$.

occupied pins and one unoccupied one. Hence, despite the increase in energy associated with the straight segments of domain wall, domain formation is favoured because the incorporation of the mismatched vortices/vacancies into domain wall corners lowers the overall energy.

\section{Theoretical models}

Fig. 4(a) and (b) illustrate some possible domain structures. Simple "book keeping" reveals that the square domain in Fig. 4(a) with four highdensity corners can accommodate exactly one excess vortex (i.e. $\Phi_{0} / 4$ per corner). Translating the square domain one lattice site vertically (or horizontally) as shown in Fig. 4(b) generates a domain with low-density corners which can accommodate exactly one vacancy. While it is not yet clear why this configuration is energetically preferred it is already evident that this represents an elegant solution to the matching problem as each domain is commensurate with the pinning array yet the mean induction averaged over several domains can vary about $\mu_{0} H_{1} / 2$.
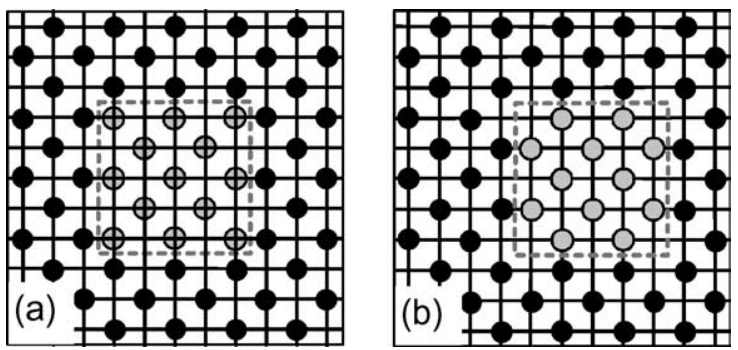

Fig. 4. Some possible domain structures near the half matching field. (a) Traps one excess vortex at the four corners, while (b) traps one vacancy.

A semi-quantitative understanding of the problem can be obtained from a simple 'meanfield' analytic model of the system, where the flux associated with each lattice site is averaged over the four adjacent unit cells. Remarkably we find that the domain walls vanish within this scheme and the only long-range interactions are between fractional flux quanta $\left(\Phi_{0} / 4\right)$ at the corners. Adding the domain wall energy ( $\sigma$ per lattice site) and assuming $w>\Lambda>a_{0}$, the total energy associated with a domain of side length $w$ is approximately $E_{\mathrm{tot}}(w) \sim 4 \sigma w / a_{0}+(4+\sqrt{2}) \Phi_{0}^{2} /\left(8 \mu_{0} \pi^{2} w\right)$. For typical sample parameters we calculate numerically that $\sigma \sim U^{\mathrm{vv}}\left(a_{0}\right) / 50 \sim \Phi_{0}^{2} / 50 \pi \mu_{0} \Lambda$ and the domain energy has a minimum at $w \sim 2 \sqrt{a_{0} \cdot \Lambda}$ as illustrated in Fig. 5. Within this mean-field picture the system can be considered to have lowered its interaction energy by breaking excess

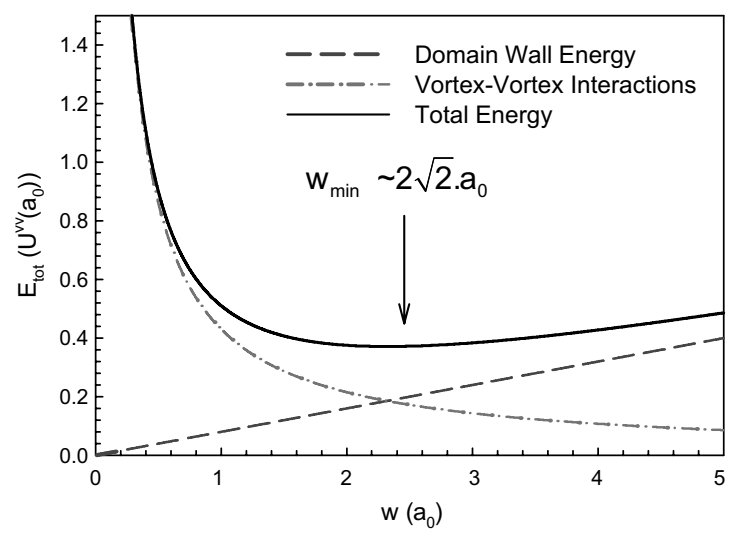

Fig. 5. Energy of a square domain near the half-matching field as a function of domain size for $\Lambda=2 a_{0}$. 
vortices or vacancies up into fractional flux quanta situated at the domain wall corners.

To demonstrate more rigorously that longrange vortex-vortex interactions promote domain formation molecular dynamics simulations were performed with a generic pinning potential. The interaction of a vortex with each pinning site was assumed proportional to $-1 /\left(\rho^{2}+c\right)$ where $\rho$ was the distance to the center of the pin, and $c^{-1}$ a measure of the pin's depth (the results did not depend sensitively on this model). Considering vortices as stiff massless lines, overdamped Langevin dynamics simulations of an effectively two-dimensional vortex system were carried out [18]. The long-range vortex-vortex interaction energy $(\lambda(T) \gg t)$ was described by [15]

$U^{\mathrm{vv}}(r)=\frac{2 \Phi_{0}^{2} t}{4 \pi \mu_{0} \lambda(T)^{2}}\left(H_{0}\left(\frac{r}{\Lambda}\right)-Y_{0}\left(\frac{r}{\Lambda}\right)\right)$

where $H_{0}\left(Y_{0}\right)$ is a Struve(Bessel) function of the second kind. The short-range vortex-vortex interaction energy, which is appropriate in thick films $(t \gg \lambda(T))$, was described by

$U^{\mathrm{vv}}(r)=\frac{2 \Phi_{0}^{2} t}{4 \pi \mu_{0} \lambda(T)^{2}} K_{0}\left(\frac{r}{\lambda}\right)$

where $K_{0}$ is the modified Bessel function of the second kind.

The differential equation of motion was solved numerically subject to periodic boundary conditions in order to compute the time progression of the system. Starting from a high temperature molten vortex configuration, we slowly annealed the system in the presence of a periodic pinning potential $\left(a_{0}=1 \mu \mathrm{m}\right)$ to zero temperature. Fig. 6(a)-(c) show typical results of the simulation for 400 pinning sites when the long-range interaction (Eq. (1)) with $\Lambda=2 \mu \mathrm{m}$ was used. For exactly half-matching conditions the checkerboard structure of Fig. 6(b) is reproduced. With one vacancy present, a rectangular domain with four low-density corners is created (Fig. 6(a)), while with one excess vortex a square domain with four highdensity corners is found (Fig. 6(c)). Fig. 6(d) is a repeat of the calculation with one excess vortex for the short-range vortex-vortex potential (Eq. (2)) where we find, as expected, that the excess vortex is

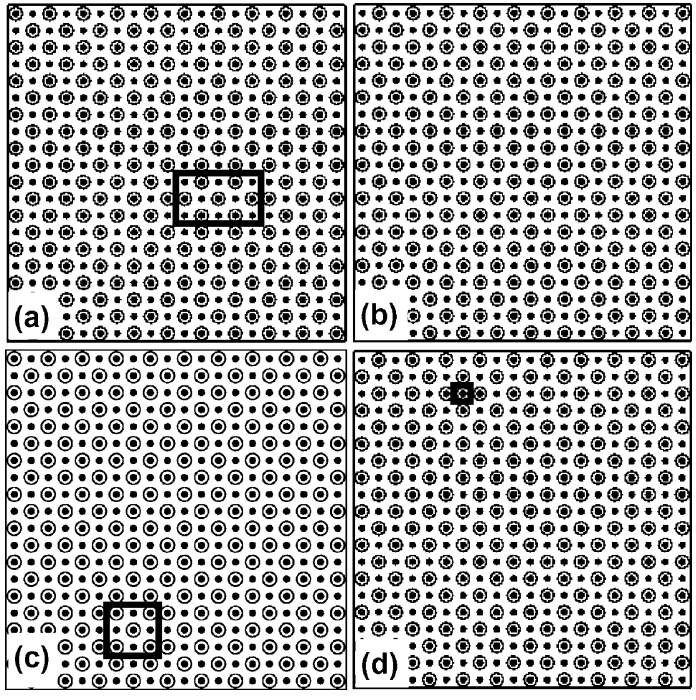

Fig. 6. Results of molecular dynamics simulations for (a)-(c) a long-range potential with $\Lambda=2 a_{0}$ and (d) for a short-range potential (see text for conditions).

located at an unoccupied pin in the checkerboard lattice.

The checkerboard structure at exactly $|H|=H_{1} / 2$ appears to be rather robust with respect to the specific form of inter-vortex interactions, as it preserves the four-fold rotational symmetry of the pinning array. This is not true of the structure at $|H|=H_{1} / 3$, where the ground state was originally predicted to be one of six degenerate symmetry-breaking chain states as sketched in Fig. 7(a) [19]. One might anticipate
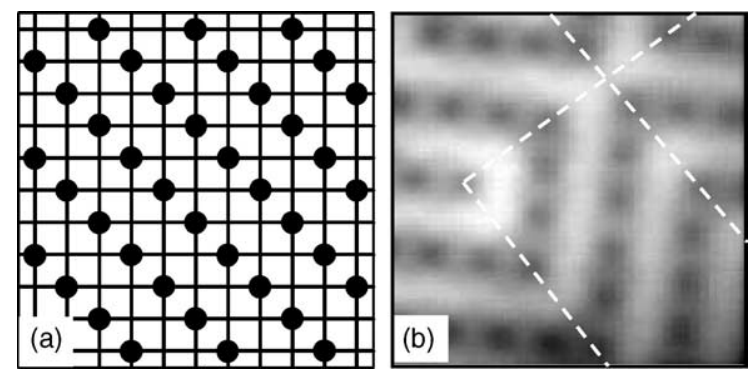

Fig. 7. (a) Predicted and (b) measured ( $T=5.5 \mathrm{~K})$ commensurate vortex structures at $H=H_{1} / 3$. The dashed white lines in (b) indicate the location of domain walls. 
that a multi-domain state would have lower energy, even exactly at the matching field, since the chain state clearly represents an inefficient way of packing vortices [20]. The multi-domain SHPM image captured at $H=-H_{1} / 3$ (Fig. 7(b)) shows this to be the case. We have also confirmed this behaviour in the molecular dynamics simulation with the same long-range vortex-vortex potential as before.

\section{Conclusions}

In conclusion, we have shown that commensurate vortex domain formation near rational fractional matching is a direct consequence of long-range vortex-vortex interactions in thin superconducting films at high temperatures. Domain wall corners incorporate fractions of excess vortices/vacancies and the overall energy is lowered because the system is able to lock to the symmetry of the pinning array within a domain, yet the magnetic induction averaged over several domains can vary around the exact matching field. This explains the relatively weak magnetisation enhancements at many rational fractional matching fields, when we presume that the critical current is limited by the rather 'easy' propagation (and nucleation) of domain walls. Matching structures, which strongly break the symmetry of the pinning array, can even be unstable with respect to domain formation exactly at the matching field. We have shown that the characteristic domain size is given by $w \sim 2 \sqrt{a_{0} \cdot \Lambda(T)}$ (where $\Lambda(T)$ is the effective penetration depth at which the domain structure becomes frozen in upon cooling below $T_{\mathrm{c}}$ ), although there will be corrections to this estimate for high concentrations of mismatched vortices.

\section{Acknowledgements}

This work was supported in the UK by EPSRC and MOD Grant No. GR/L96448. The authors thank S. Raedts, L. Van Look, K. Temst, J. Bekaert and R. Jonckheere for help with sample preparation and C.J. Olson and C. Reichhardt for valuable discussions. This work was supported by the Belgian Inter-University Attraction Poles (IUAP) and Flemish Concerted Research Actions (GOA) programs, by the ESF "VORTEX" program and by the Fund for Scientific ResearchFlanders (FWO). MJVB is a Postdoctoral Research Fellow of the FWO.

\section{References}

[1] A.F. Hebard, A.T. Fiory, S. Somekh, Appl. Phys. Lett. 32 (1978) 73.

[2] M. Baert et al., Phys. Rev. Lett. 74 (1995) 3269.

[3] M. Baert et al., Europhys. Lett. 29 (1995) 157.

[4] J.I. Martín et al., Phys. Rev. Lett. 79 (1997) 1929.

[5] M.J. Van Bael et al., Phys. Rev. Lett. 86 (2001) 155.

[6] D.J. Morgan, J.B. Ketterson, Phys. Rev. Lett. 80 (1998) 3614.

[7] M.J. Van Bael et al., Physica C 369 (2002) 97.

[8] C. Reichhardt et al., Phys. Rev. B 54 (1996) 16108.

[9] K. Harada et al., Science 274 (1996) 1167.

[10] S.B. Field et al., Phys. Rev. Lett. 88 (2002) 067003.

[11] A.N. Grigorenko et al., Phys. Rev. B 63 (2001) 052504.

[12] L.D. Cooley, A.M. Grishin, Phys. Rev. Lett. 74 (1995) 2788.

[13] V.V. Moshchalkov et al., Phys. Rev. B 54 (1996)7385.

[14] A.N. Grigorenko et al., Phys. Rev. Lett. 90 (2003) 237001.

[15] M.J. Van Bael et al., Phys. Rev. B 68 (2003) 014509.

[16] J. Pearl, Appl. Phys. Lett. 5 (1964) 65.

[17] A. Oral, S.J. Bending, M. Henini, Appl. Phys. Lett. 69 (1996) 1324

[18] H. Fangohr, S.J. Cox, P.A.J. de Groot, Phys. Rev. B 64 (2001) 064505.

[19] G.I. Watson, Physica (Amsterdam) 246A (1997) 253.

[20] C. Reichhardt, N. Gronbech-Jensen, Phys. Rev. B 63 (2001) 054510. 